\title{
A survey of Midwest physicians' experiences with patients in psychiatric distress in the emergency department
}

https://doi.org/10.1515/jom-2021-0052

Received February 14, 2021; accepted June 8, 2021;

published online July 27, 2021

\section{Abstract}

Context: Emergency medicine (EM) physicians commonly stabilize patients with acute psychiatric distress, such as suicidal ideation. Research has shown that suicidal ideation is difficult to manage in emergency department (ED) settings and that patients in psychiatric distress are often "boarded" in the ED while awaiting more definitive care.

Objectives: To examine the attitudes and experiences of emergency physicians regarding the care of patients in psychiatric distress. Special attention is given to suicidal ideation due to its prevalence in the United States.

Methods: A 19 question anonymous survey was sent via email to 55 emergency medicine residency directors throughout Michigan, Ohio, Indiana, and Illinois, who were identified using an Internet search of residency programs in the region. The program directors were asked to distribute the survey to their colleagues and residents. The intent of this procedure was to generate as many survey responses as possible, while obscuring the identities of the respondents. Responses were gathered from October 29, 2019 until January 16, 2020. The survey was designed to assess respondents' self-reported demographic data as well as their experiences with the boarding process, initial examination, final disposition, reevaluation of the patient,

*Corresponding author: Jack Brodeur, BS, OMS III, Michigan State University College of Osteopathic Medicine, 965 Wilson Road, Suite A233, East Lansing, MI, 48824, USA, E-mail: brodeur5@msu.edu. https://orcid.org/0000-0002-0390-2144

Alyse Folino Ley, DO, Psychiatry Residency Program, Department of Psychiatry, Michigan State University College of Osteopathic Medicine, East Lansing, MI, USA; and Child and Adolescent Psychiatry Fellowship, Department of Psychiatry, Michigan State University College of Osteopathic Medicine, East Lansing, MI, USA

Michelle Bonnet, MD, MBA, Psychiatry Residency Program, Michigan State University College of Osteopathic Medicine, East Lansing, MI, USA

Ә Open Access. @ 2021 Jack Brodeur et al., published by De Gruyter. (c) BY International License. physician training and resources, and follow up care. Statistical analysis was performed using a Mann-Whitney $\mathrm{U}$ test, significance was set at $\mathrm{p}<0.01$.

Results: In total, $47 \mathrm{EM}$ physicians responded to the survey; however, not all of the respondents completed all 19 questions. Ten of 44 respondents (22.7\%) reported that they do not perform the initial psychiatric examination themselves and instead defer to a nurse or social worker. Twentytwo of 44 respondents (50.0\%) reported that they defer to a social worker when determining the final disposition of psychiatric patients. Respondents reevaluated patients in psychiatric distress statistically significantly less often $(\mathrm{p}=0.01)$ compared with patients with cardiac pathology. Additionally, 15 of 38 respondents (39.5\%) reported that they did not feel adequately trained to handle psychiatric emergencies, and 36 of 39 respondents (92.3\%) of physicians felt that their facility would benefit from additional mental health resources. Thirty five of 39 respondents (89.7\%) reported that their facility did not have a system in place to follow up with suicidal patients upon discharge. Conclusions: Caring for patients who are acutely suicidal or in psychiatric distress is complex and more research is needed to optimize treatment strategies. The results of this study indicate that EM physicians may regularly defer to nonphysician providers when evaluating and treating patients in psychiatric distress. A perceived lack of training in psychiatry may contribute to this practice. The results of this study are in accord with previous research that indicated a need for additional psychiatry training in EM residencies.

Keywords: clinical practice; emergency medicine; psychiatric distress; psychiatry; suicidal ideation.

Individuals with psychiatric complaints can be managed in all medical settings, but psychiatric emergencies are often treated and stabilized in the emergency department (ED). Approximately one of every eight patients presenting to the ED has a mental health concern [1]. Emergency medicine (EM) physicians are responsible for the care, evaluation, and management of psychiatric patients, including those with acute suicidal ideation when they present to the ED. 
Managing a suicidal patient in the ED can be challenging. Suicide is the second leading cause of death in Americans aged 10-24 years [2] and is the 10th leading cause of death in the United States [3]. Despite extensive research, it remains difficult for physicians to accurately predict suicidality, and ED interventions for acutely suicidal patients remain limited [4,5]. Studies [6-8] have consistently demonstrated that a high percentage of individuals who die from suicide received care in an emergency department (ED) in the year prior to their death.

The current standard of care for patients presenting to an ED with suicidal ideation includes the following: ensure medical stability, evaluate for underlying and contributing medical illness, and finally, determine whether the patient is at risk of harm and whether they should be involuntarily held until psychiatric hospitalization is possible [4]. A 1 year, single center study of 251 patients found that suicidal ideation accounted for the majority of involuntary psychiatric holds (73.7\%) in the ED [9]. Some patients are retained in the ED for more than 24 hours and, in extreme cases, longer than 7 days; this practice commonly is referred to as ED "boarding." [10] Boarding in the ED can impose significant stress on the patient and excessive strain on resources. In a 2008 study by the American College of Emergency Physicians, $62 \%$ of 328 emergency department directors reported that there were no psychiatric resources available for patients while they were being boarded [10]. Although the boarded patients are kept safe, they are unlikely to receive other meaningful treatment during their stay in the ED. Furthermore, previous studies $[10,11]$ have confirmed that psychiatric boarding places significant demands on ED staff and resources and negatively impacts ED wait times.

Although the stress generated on EDs by boarding psychiatric patients is well documented, research about physicians' views regarding the care of psychiatric patients in the ED has been limited. To better understand the perspectives of emergency physicians, our research team designed an online survey to assess their attitudes and experiences of emergency medicine physicians who manage patients in psychiatric distress in an ED setting.

\section{Methods}

This study was reviewed and deemed exempt category by the Michigan State University Institutional Review Board (MSU Study ID: STUDY00003520).

A 19 question anonymous survey (Supplementary Material) was sent via email to 55 emergency medicine residency directors throughout Michigan, Ohio, Indiana, and Illinois who were identified using an Internet search of residency programs in the region. Recipient program directors were asked to distribute the survey to their colleagues and residents. Responses were gathered from October 29, 2019 until January 16, 2020. The survey was administered using Qualtrics XM.

The intent of our data collection procedure was to use snowball sampling to generate as many survey responses as possible, while obscuring the identities of the respondents. As a result, certain demographic information was not collected, including the experience level of respondents (i.e., resident vs. attending) and the number of respondents from each individual residency program. Additionally, our survey tool was not capable of tracking responses and there were no linked questions allowing for attributed responses between earlier and later questions.

The survey (Supplementary Material) was developed by the authors. The survey was designed to assess respondents' self-reported demographic data as well as their experiences with the boarding process, initial examination, final disposition, reevaluation of the patient, physician training and resources, and follow up care.

All respondents were notified that their responses to the survey indicated their consent to participate in research. Statistical analysis was performed using a Mann-Whitney U test [12, 13].

\section{Results}

\section{Response rate and self-reported demographics}

Forty seven emergency medicine physicians responded to the survey, 38 (80.9\%) of whom responded to all questions (some questions received less than 47 but greater than 38 responses). Twenty seven of 47 respondents (57.4\%) selfidentified as working in academic hospitals and 20 (42.6\%) reported working in community hospitals. Additionally, 11 of $46(23.9 \%)$ reported that they work at a facility with a designated psychiatric ED or unit. Twenty-five of 47 respondents (53.2\%) were doctors of osteopathic medicine (DOs) and 22 (46.8\%) were doctors of medicine (MDs). Four of 45 respondents $(8.9 \%)$ stated that their EDs are staffed by psychiatrists.

\section{Initial examination}

Thirty four of 44 respondents (77.3\%) reported that they perform the initial psychiatric examination on their patients, while $10(22.7 \%)$ reported that this examination was carried out by either a nurse or a social worker (Figure 1A). Ten of 44 respondents (22.7\%) reported that if they do not perform the initial psychiatric examination, they also do not perform their own examination to check the work of the nurse or social worker. Our survey did not differentiate between registered nurses (RNs) and nurse practitioners (NPs); we only provided options for "nurse" and "midlevel provider" on the survey among the options to answer the 
(A)

\section{INITIALEXAM}

a Physician $\quad$ Nurse/Social Worker

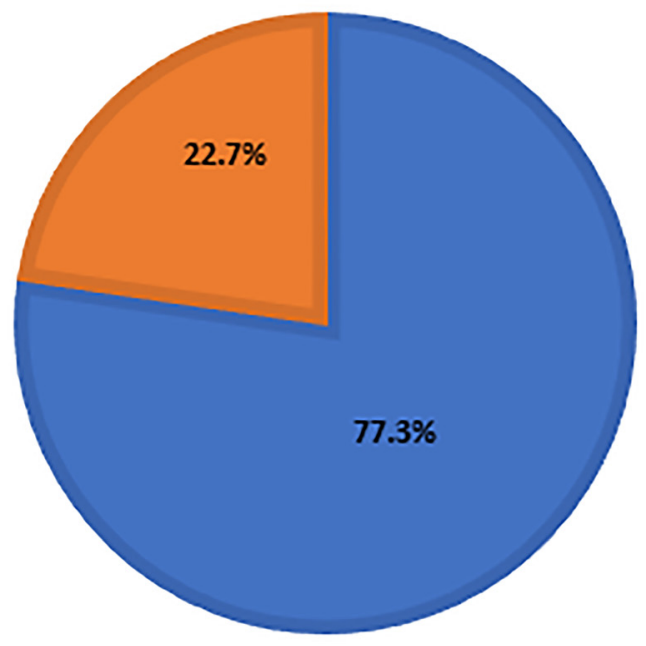

(B)

\section{FINALDISPOSITION}

Physician Social Worker Midlevel/Nurse Psychiatrist

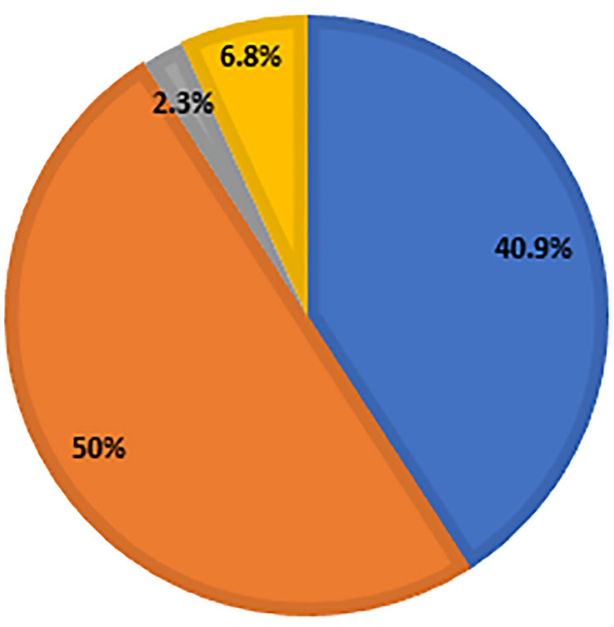

Figure 1: Respondents' reported provider evaluation patterns for (A) initial psychiatric patient examination and (B) determination of final patient disposition.

question "Who performs the initial psychiatric evaluation of a suicidal patient?" We did not provide a specific definition of midlevel providers, a term assumed to encompass NPs and physician assistants (PAs). Regardless, no respondents reported using a midlevel provider to perform the initial evaluation of ED patients in psychiatric distress.

\section{Final disposition}

18 of 44 respondents (40.9\%) reported that they determine the final disposition of psychiatric patients (Figure 1B). Of those, $22(50.0 \%)$ reported that a social worker determines the final disposition. One physician $(2.3 \%)$ reported that a midlevel provider determines the final disposition. Three respondents $(6.8 \%)$ stated that a psychiatrist determines the final disposition; due to the aforementioned limitations in data collection, it is unclear whether those three physicians work at three different EDs and whether their EDs have separate psychiatric departments.

\section{Reevaluation}

Twenty of 44 respondents (45.5\%) reported that they reevaluate suicidal patients once per shift while the patient is being boarded in the ED and 14 (31.8\%) reported that they do not reevaluate boarded patients at all (Figure 2). For comparison, respondents were asked about patients being boarded in the ED with cardiac pathology. Sixteen of 41 respondents $(39.0 \%)$ reported reevaluating patients with cardiac pathology once every $2 \mathrm{~h}$, while 11 of 41 respondents (26.8\%) reported reevaluating them once every $4 \mathrm{~h}$; eight of $41(19.5 \%)$ reported that they do not reevaluate patients with cardiac pathology during the course of ED boarding. Based on this data, respondents reevaluated patients in psychiatric distress statistically significantly less often $(\mathrm{p}=0.01)$ compared with patients with cardiac pathology. However, this comparison between reevaluation patterns among respondents in their care of suicidal vs. cardiac patients in the ED is limited because the survey did not examine how often EM physicians utilized consultants for each group of patients; as lack of consultant support could significantly impact the frequency of reevaluation.

\section{Physician training and resources}

Fifteen of 38 respondents (39.5\%) indicated that they did not feel they were adequately trained to handle psychiatric emergencies such as suicidal ideation. Thirty six of 39 respondents $(92.3 \%)$ reported that their facility would benefit from additional mental health resources. Thirty five of 39 respondents $(89.7 \%)$ reported that their facilities do not have systems in place to follow up with suicidal patients upon discharge. 


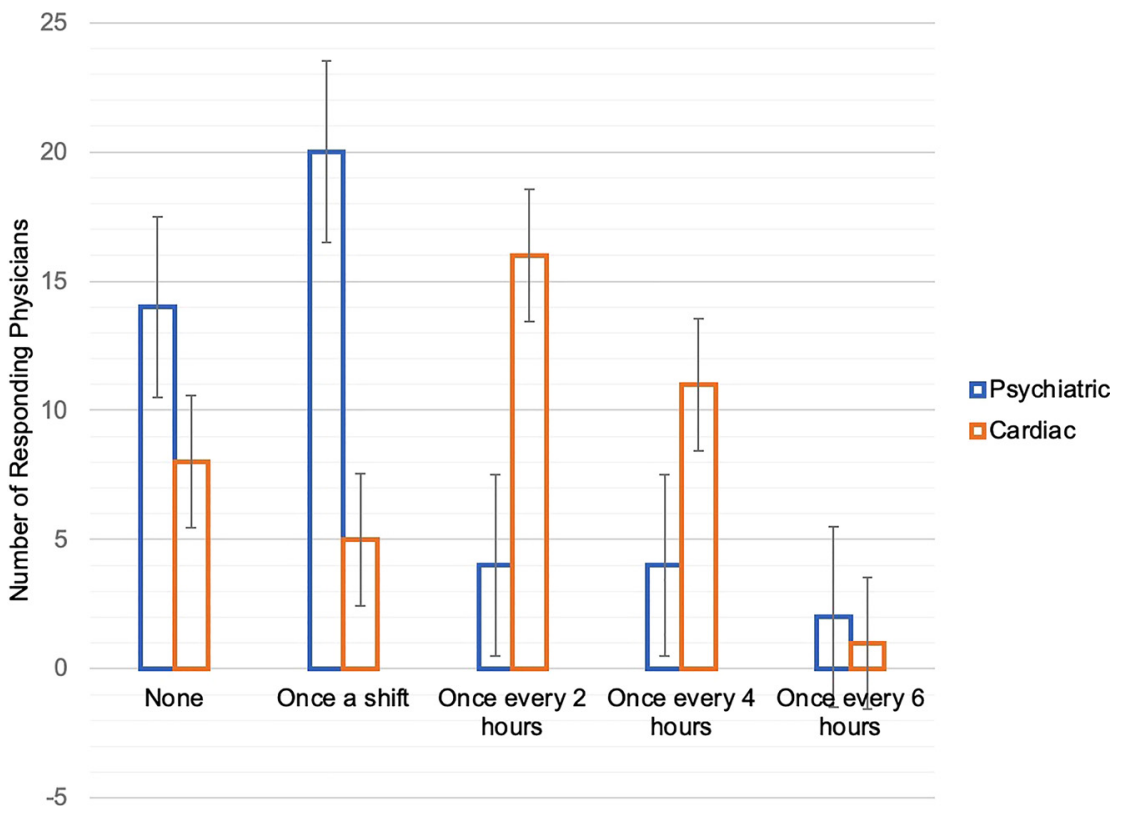

Figure 2: Respondents' reported frequency of reevaluation of patients who are being boarded in the ED. Mean frequency of evaluation for a suicidal patient was once per shift compared with a mean frequency of evaluation once every $4 \mathrm{~h}$ for cardiac patients $(p=0.01)$.

\section{Discussion}

Although the majority of EM physician respondents in our study reported that they perform the initial psychiatric evaluation on their patients, $26.6 \%$ did not; the reasons EM physicians may defer the initial psychiatric examination of their patients are likely multifactorial and deserves further exploration. We hypothesize that this host of factors may include access to in-house psychiatrists, lack of psychiatric training/education, and increased ED crowding. Of note, four of 45 respondents (8.9\%) did report that their ED is staffed by psychiatrists.

It is atypical for an EM physician to defer evaluation and determination of patient disposition to a nurse, social worker, or other nonphysician provider. However, our survey data indicate that this may be a fairly common practice in the treatment of psychiatric patients. According to our survey results, this could be in part due to EM physicians feeling insufficiently trained to handle psychiatric emergencies; $39.5 \%$ of our respondents indicated that they did not feel they were adequately trained in that area. This finding is supported by Bode and Jackson's 2017 study [14], which confirmed that most EM residencies lack psychiatry rotations, and that psychiatry is underrepresented on EM board examinations [14]. This sentiment was echoed in a report [11] on focus group sessions with resident and attending physicians at a large teaching hospital, in which a number of EM physicians stated their discomfort and perceived lack of qualification to assess suicidality.

Our sample of EM physicians reported that they were less likely to reevaluate psychiatric patients who are being boarded in the ED when compared with cardiac patients; 14 respondents $(31.8 \%)$ reported that they do not reevaluate psychiatric patients once boarded. This is a notable finding, as it suggests that patients with psychiatric illness may go many hours, or even days, without being reevaluated by a physician. This practice is inconsistent with the recommendations of the National Suicide Prevention Center, which advocates for physicians to treat suicidal patients the same as medical patients [15]. The National Suicidal Prevention Center's guidelines also encourage EM physicians to evaluate suicidal patients frequently during their ED stays and emphasize the importance of building rapport with these patients [15]. We did not ask how often EM physicians utilize consultants in the care of suicidal patients; routine utilization of psychiatry or psychology consultants may explain the low reevaluation rates in our study population.

A 2017 study [16] of ED interventions identified post discharge follow up as a means of reducing suicidality in patients who present with acute suicidal ideation. Further research [17] has shown that ED screening accompanied by follow up intervention via telephone may reduce suicide attempts and deaths by $10 \%$ in the year following the initial ED presentation. This follow up activity then has the potential to reduce the number of suicides and suicide attempts by 39,500 and save $\$ 5.84$ billion annually in the United States [18]. The National Suicide Prevention Center also advocates for EDs working with local crisis centers to ensure follow up for suicidal patients [15]. The results of our survey indicate that the majority (89.7\%) of the ED staffed by the respondent physicians do not have follow up 
programs in place; we believe this may be attributed to lack of mental health resources in the ED and in the community.

\section{Limitations}

The results of our study provide insight into the perceptions and experiences of ED physicians surrounding treatment of psychiatric emergencies in the Midwest United States prior to the novel coronavirus 2019 pandemic. This survey did not explore practice patterns of EM physicians who stated that their EDs had in house psychiatrists, psychiatric units, or access to separate psychiatric ED. These resources could significantly impact EM physician workflow, decision making, and their responsibility for evaluation and treatment of patients with psychiatric complaints. Additionally, the procedure we used to anonymize survey respondents rendered it impossible to know the ages and experience levels of the emergency physician respondents. It is therefore unclear whether those factors influenced responses to the survey. Furthermore, we study did not include midlevel providers or non EM physicians (i.e., family medicine and internal medicine physicians) who practice in EDs in our survey. It is also important to note that our study population was small $(\mathrm{n}=47)$, and our results should not be generalized across the entire field of EM.

\section{Conclusions}

Psychiatric care in the emergency department is challenging for EM physicians, staff, and patients. Lack of resources and professed lack of training impact the EM physician's overall confidence in treating this group of patients. While EM physicians would not normally defer the evaluation and determination of disposition for patients to a nurse, social worker, or other nonphysician provider, our survey results indicated that this may be a fairly common occurrence in the management of psychiatric patients. According to our small survey this may potentially be in part due to EM physicians feeling insufficiently trained to handle psychiatric emergencies. Our finding regarding a perception of insufficient training is supported by the fact that most EM residencies lack psychiatry rotations, and that psychiatry is underrepresented on EM board examinations.

Further research is necessary to assess whether the results of this study are consistent with EM practice nationwide and to more critically assess practice patterns in EDs with and without access to psychiatry resources.
Future studies are needed to explore barriers to care, improve physician comfort and education, and assist in the design of increased training opportunities. Increased collaboration between EM and psychiatry services may allow for the development of strategies that minimize ED boarding time for patients with suicidal ideation and psychiatric distress, as well as improve the quality of acute and follow-up care for this patient population.

Research funding: None reported.

Author contributions: All authors provided substantial contributions to conception and design, acquisition of data, or analysis and interpretation of data; Mr. Brodeur and Dr. Ley drafted the article or revised it critically for important intellectual content; all authors gave final approval of the version of the article to be published; and all authors agree to be accountable for all aspects of the work in ensuring that questions related to the accuracy or integrity of any part of the work are appropriately investigated and resolved.

Competing interests: None reported.

Ethical approval: This study was reviewed deemed exempt by the Michigan State University Institutional Review Board (MSU Study ID: STUDY00003520).

Informed consent: All participants were informed that by participating in this electronic, email-delivered survey, they were giving their consent for their answers to be used for research.

\section{References}

1. Moore BJ, Stocks C, Owens PL. Trends in emergency department visits, 2006-2014. HCUP statistical brief \#227. September 2017. Rockville, MD: Agency for Healthcare Research and Quality. https://www.hcup-us.ahrq.gov/reports/statbriefs/ sb227-Emergency-Department-Visit-Trends.pdf [Accessed 1 June 2021].

2. Heron M. Deaths: leading causes for 2017. Natl Vital Stat Rep 2019; 68:1-77.

3. Xu J. Mortality in the United States, 2018. NCHS data brief, no. 355. Hyattsville, MD: National Center for Health Statistics; 2020.

4. Nazarian DJ, Broder JS, Thiessen ME, Wilson MP, Zun LS, Brown $M D$, et al. Clinical policy: critical issues in the diagnosis and management of the adult psychiatric patient in the emergency department. Ann Emerg Med 2017;69:480-98.

5. Betz ME, Boudreaux ED. Managing suicidal patients in the emergency department. Ann Emerg Med 2016;67:276-82.

6. Gairin I, House A, Owens D. Attendance at the accident and emergency department in the year before suicide: retrospective study. Br J Psychiatr 2003;183:28-33.

7. Da Cruz D, Pearson A, Saini P, Miles C, While D, Swinson N, et al. Emergency department contact prior to suicide in mental health patients. Emerg Med J 2011;28:467-71. 
8. Ahmedani BK, Simon GE, Stewart C, Beck A, Waitzfelder BE, Rossom R, et al. Health care contacts in the year before suicide death. J Gen Intern Med 2014;29:870-7.

9. Roy A, Lachner C, Dumitrascu A, Dawson NL, Vadeboncoeur TF, Maniaci M, et al. Patients on involuntary hold status in the emergency department. South Med J 2019;112:265-70.

10. ACEP Psychiatric and substance abuse survey 2008. https:// www.acep.org/globalassets/uploads/uploaded-files/acep/ advocacy/federal-issues/psychiatricboardingsummary.pdf [Accessed 23 June 2021].

11. Roy W, Roaten K, Downs D, Khan F, Pollio DE, North CS. Suicide risk assessment and management: real-world experience and perceptions of emergency medicine physicians. Arch Suicide Res 2017;21:365-78.

12. Bruning JL, Kintz BL. Computational handbook of statistics. Glenview, IL: Scott, Foresman, and Company; 1968.

13. Fay MP, Malinovsky Y. Confidence intervals of the Mann-Whitney parameter that are compatible with the Wilcoxon-Mann-Whitney test. Stat Med 2018;37:3991-4006.
14. Bode A, Jackson JS. The current emergency medicine residency curriculum: missing psychiatry. Am J Emerg Med 2017;35:1771-2.

15. Suicide Prevention Resource Center. Caring for adult patients with suicide risk: a consensus guide for emergency departments. Waltham, MA: Education Development Center, Inc.; 2015.

16. Miller IW, Camargo CA, Arias SA, Sullivan AF, Allen MH, Goldstein $A B$, et al. Suicide prevention in an emergency department population: the ED-SAFE study. JAMA Psychiatry 2017;74:563-70.

17. Dunlap LJ, Orme S, Zarkin GA, Arias SA, Miller IW, Camargo CA Jr, et al. Screening and intervention for suicide prevention: a costeffective analysis of the ED-SAFE interventions. Psychiatr Serv 2019;70:1082-7.

18. Shepard DS, Gurewich D, Lwin AK, Reed GA Jr, Silverman MM. Suicide and suicidal attempts in the United States: costs and policy implications. Suicide Life Threatening Behav 2016;46:352-62.

Supplementary Material: The online version of this article offers supplementary material (https://doi.org/10.1515/jom-2021-0052). 